\title{
Land Cover Classification of Remotely Sensed Satellite Data using Bayesian and Hybrid classifier
}

\author{
${ }^{1}$ Ratika Pradhan, ${ }^{2}$ M. K. Ghose, ${ }^{3}$ A. Jeyaram \\ ${ }^{1,2}$ Department of Computer Science and Engineering, Sikkim Manipal Institute of Technology, \\ Rangpo, Sikkim, INDIA. \\ ${ }^{3}$ Regional Remote Sensing Service Centre, ISRO, IIT Campus, \\ Kharagpur, INDIA
}

\begin{abstract}
In this paper an attempt has been made to develop classification algorithm for remotely sensed satellite data using Bayesian and hybrid classification approach. Bayesian classification is a probabilistic technique which is capable of classifying every pattern until no pattern remains unclassified. Hybrid classification involves developing training patterns using unsupervised classification followed by classifying the pixels using supervised classification. It is observed that the overall accuracy was found to be $90.53 \%$ using the Bayesian classifier and $91.57 \%$ using the Hybrid classifier.
\end{abstract}

\section{Keywords}

Bayesian classifier, Hybrid classifier, K-means, confusion matrix, overall accuracy.

\section{INTRODUCTION}

Classification is the task of assigning a set of given data elements to a given set of labels or classes. The major steps of image classification may include determination of a suitable classification system, selection of training samples, image preprocessing, and feature extraction and selection of suitable classification approaches. There are three basic classification strategies:

- $\quad$ Supervised Classification techniques require training areas to be defined by the analyst in order to determine the characteristics of each category

- Unsupervised Classification searches for natural groups of pixels, called clusters, present within the data by means of assessing the relative locations of the pixels in the feature space

- Hybrid Classification: It takes the advantage of both the supervised classification and unsupervised classification.

Traditionally, classification tasks are based on statistical methodologies such as Minimum Distance-to-Mean (MDM), Maximum Likelihood Classification (MLC) and Linear Discrimination Analysis (LDA). These classifiers are generally characterized by having an explicit underlying probability model, which provides a probability of being in each class rather than simply a classification. The performance of this type of classifier depends on how well the data match the pre-defined model. If the data are complex in structure, then to model the data in an appropriate way can become a real problem.

\section{RELATED WORK}

There exist various classification methods for classifying satellite imagery which includes Maximum likelihood classifier, $\mathrm{k}-\mathrm{NN}$ classifier, k-means classifier, parallel piped classifier, decision tree, ANN classifer and fuzzy classifier. A. Tzotsos and D. Argialas, has suggested Support Vector Machine classification technique for Object Based Image Analysis (OBIA) for supervised classification of the satellite imagery using object based representation [1]. X. Gigandet et.al. have suggested region based satellite image classification that combines unsupervised segmentation with supervised segmentation using Gaussian hidden Markov random field and Support Vector Machine [2]. Friedl and Brodley [3] have used decision tree classification method to classify land cover using univariate, multivariate and hybrid decision tree as the base classifier and found that hybrid decision tree outperform the other two. Mahesh Pal and Paul M. Mather [4], [5] have suggested boosting techniques for the base classifier to classify remotely sensed data to improve the overall accuracy. B. Gorte and A. Stein [6] have used Bayesian classification for class area estimation of satellite images using stratification. LAU C.C. and Hsiao K.H. [7] have proposed Bayesian Classifier for rice paddy interpretation. They have presented a case study of interpreting paddy distributions of three counties on Northern Taiwan during two crop seasons on year 2000 using multitemporal imageries together with cadastre GIS by Bayesian posteriori probability classifier. Jeliazkova $\mathrm{N}$. et al. [8] have proposed a nonparametric Bayesian classification, based on very fast algorithm for multivariate density estimation for recognition of land cover type of remotely sensed data. Perea1 J. A. et al. [9] developed a methodology for the classification of digital aerial images, which, with the aid of object-based classification and the Normalized Difference Vegetation Index (NDVI), can quantify agricultural areas, by using algorithms of expert classification, with the aim of improving the final results of thematic classifications. It has been shown in [10] by Besag et al. that Bayesian models often result in maps of far superior quality.

\section{BAYESIAN CLASSIFICATION}

Bayesian methods take the parameters as random variables with known prior distribution. It is based on Bayes' Classifier. A Bayes classifier assumes that the presence (or absence) of a particular feature of a class is unrelated to the presence (or absence) of any other feature. It estimates the probabilities of occurrence of different attribute values for the different classes 
in a training set. It then uses these probabilities to classify recall patterns.

Let

- $\quad$ A be an array of $M \geq 1$ attributes $A_{1}, A_{2}, \ldots ., A_{M}$ for the patterns of a training set, and

- $\quad \mathbf{P}\left(\mathbf{A}^{*}\right)$ is the probability that a training pattern has attribute array $\mathrm{A}^{*}$, regardless of the class to which the pattern belongs, the attributes having discrete values.

Suppose the training set has patterns from $m \geq 1$ classes $C_{1}, C_{2} \ldots$ $\mathrm{C}_{\mathrm{m}}$. For $1 \leq \mathrm{k} \leq \mathrm{m}$, we define the following probabilities.

- $\quad \mathrm{P}\left(\mathrm{C}_{\mathrm{k}}\right)$ is the probability that a training pattern belongs to class $\mathrm{C}_{\mathrm{k}}$. It is also known as the prior probability of class $\mathrm{C}_{\mathrm{k}}$.

- $\quad \mathrm{P}\left(\mathrm{C}_{\mathrm{k}} \mid \mathrm{A}^{*}\right)$ is the probability that a training pattern with attribute array $A^{*}$ belongs to class $C_{k}$. this is also known as the posterior probability of $\mathrm{C}_{\mathrm{k}}$ for a given $\mathrm{A}^{*}$. The attributes have discrete values.

- $\quad \mathrm{P}\left(\mathrm{A}^{*} \mid \mathrm{C}_{\mathrm{k}}\right)$ is the conditional probability that a training pattern of class $C_{k}$ has attribute array $A^{*}$, the attributes having discrete values.

- $\quad \mathrm{p}\left(\mathrm{A}^{*} \mid \mathrm{C}_{\mathrm{k}}\right)$ is the conditional probability density that a training pattern of class $\mathrm{C}_{\mathrm{k}}$ has attribute array $\mathrm{A}^{*}$, the attributes having continuous values.

According to the Bayes' theorem of probability theory

$$
\mathrm{P}\left(\mathrm{C}_{\mathrm{k}} \mid \mathrm{A}^{*}\right)=\frac{P\left(C_{k}\right) P\left(A^{*} \mid C_{k}\right)}{P\left(A^{*}\right)}
$$

Suppose the number of patterns in class $\mathrm{Ck}$ is $|\mathrm{Ck}|$, for $1 \leq \mathrm{k} \leq$ $m$. Then under the conventional estimation known as the maximum - likelihood estimation,

$$
P\left(C_{k}\right)=\frac{\left|C_{k}\right|}{\sum_{j=1}^{m}\left|C_{j}\right|}
$$

Under the alternative estimation, called the Bayesian estimation,

$$
P\left(C_{k}\right)=\frac{\left|C_{k}\right|+1}{m+\sum_{j=1}^{m}\left|C_{j}\right|}
$$

In the Bayesian estimation, we assume the number of patterns of each class to be one more than actually present in the training set. For a typical training set with a lot of patterns (that is, $\sum_{\mathrm{j}=1}^{\mathrm{m}}|\mathrm{Cj}|$ is a large number), both the maximum likelihood and Bayesian estimates give approximately equal values. Moreover for both estimates, $\sum_{\mathrm{k}=1}^{\mathrm{m}} \mathrm{P}\left(\mathrm{C}_{\mathrm{k}}\right)=1$, as it should be. One difference exists between the two estimations: in the maximum likelihood estimation, $\mathrm{P}(\mathrm{Ck})>0$. In other words, in the Bayesian estimation a probability is never equal to zero.

\section{HYBRID CLASSIFICATION}

Hybrid Classification includes the advantages of both supervised as well as unsupervised classification. We implemented hybrid classification to refine the results obtained from Bayesian Classification. For unsupervised classification, K-means clustering algorithm is used followed by Bayesian Method for supervised classification.
$\mathrm{K}$-means is one of the simplest unsupervised learning algorithms that solve the well known clustering problem. The procedure follows a simple and easy way to classify a given data set through a certain number of clusters (assume $\mathrm{k}$ clusters) fixed a priori. This main idea is to define $\mathrm{k}$ centroids, one for each cluster. These centroids should be placed in cunning way because different locations cause different results. So, the better choice is to place them as far away as possible from each other. The next step is to take each point belonging to a given data set and associate it to the nearest centroid. When no point is pending, the first step is completed and an early grouping is done. At this point we need to re-calculate $\mathrm{k}$ new centroids of the clusters resulting from the previous step. After we have these $\mathrm{k}$ new centroids, a new binding has to be done between the same data set points and the nearest new centroid. A loop has been generated. As a result of this loop we may notice that the $\mathrm{k}$ centroids change their locations step by step until no more changes are done. In other words, centroids do not move any more.

Steps Involved in K-means clustering:

Step 1. Place K points into the space represented by the object that is being clustered. These points represent initial group centroids.

Step 2. Assign each object to the group that has the closest centroid.

Step 3. When all the objects have been assigned, recalculate the positions of the $\mathrm{k}$ centroids.

Step 4. Repeat steps 2 and 3 until the centroids no longer move. This produces a separation of the objects into groups from which the metric to be minimized can be calculated [11].

\section{RESULTS AND DISCUSSIONS}

To validate the applicability of the proposed Bayesian and Hybrid Classifier, a case study is presented in this section, which is carried out on IRS-1C/LISS III sample image with $23 \mathrm{~m}$ resolution. The FCC (False Color Composite) of the input image (Figure 1) belongs to East Sikkim, India and band used to prepare FCC includes - Red (R), Green (G), Near Infrared (NIR). The IRS-IC/LISS III data of East Sikkim was acquired on October, 2006. The input image is shown in Figure 1. Figure 2 shows the result of applying Bayesian Classifier and figure 3 shows the result of applying Hybrid Classifier. In hybrid classifer, K-means clustering algorithm is used first to refine the training patterns. These refined training patterns were given as input to the Bayesian Classification Algorithm and the results thus obtained are an improvement over the previous method. 


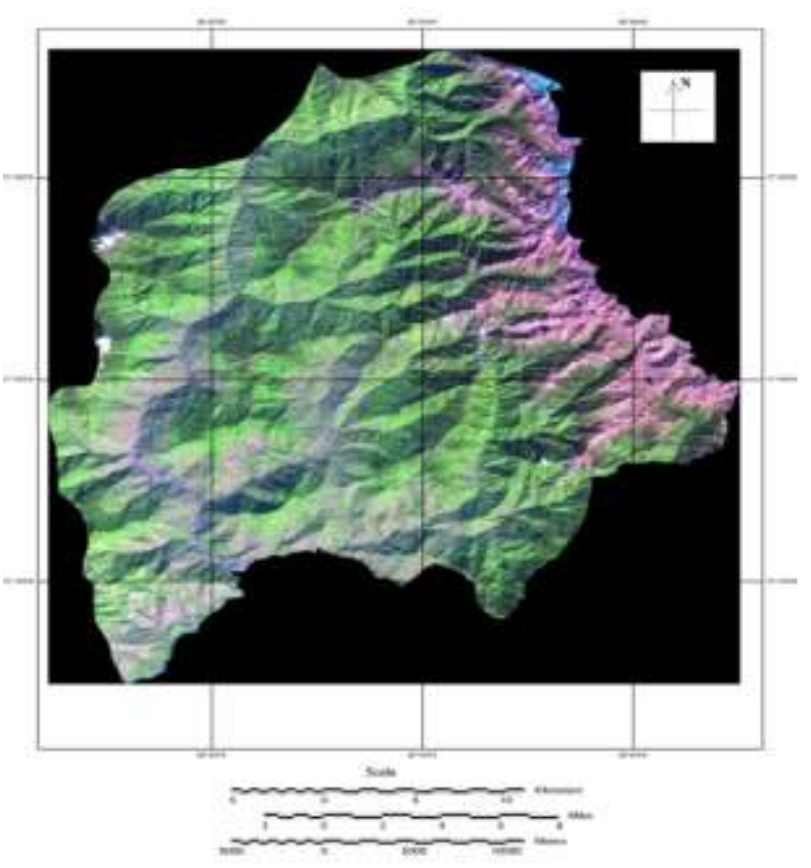

Figure 1: FCC (IRS LISS III Image -2006) of study area East Sikkim, INDIA, Band used - Red (R), Green (G), Near Infrared (NIR).

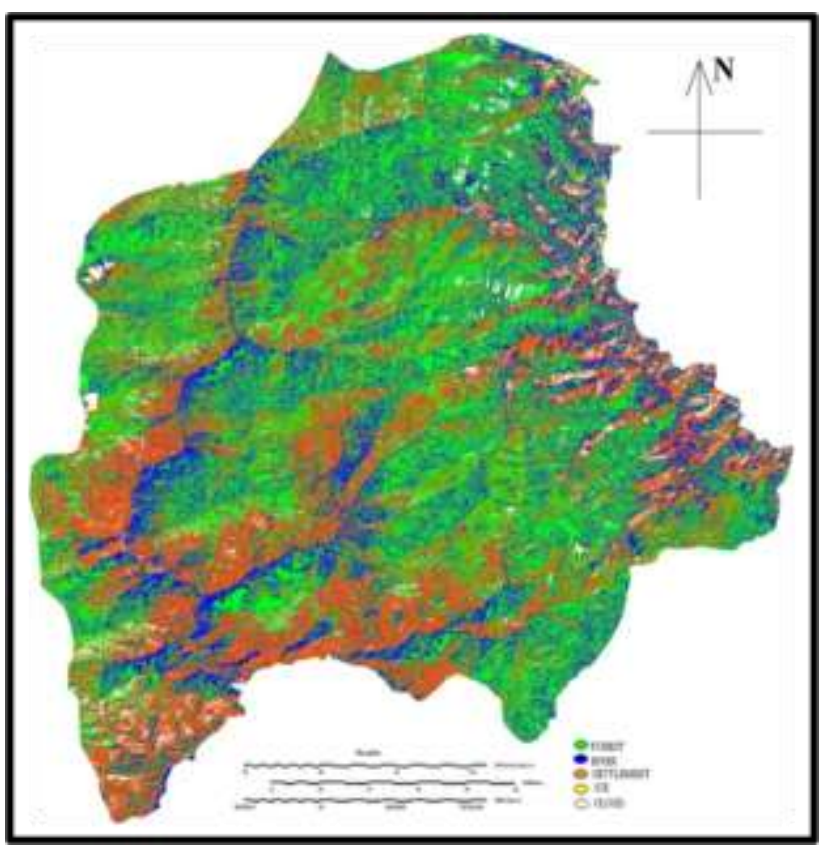

Figure 2: Classified image based on Bayesian classifier.

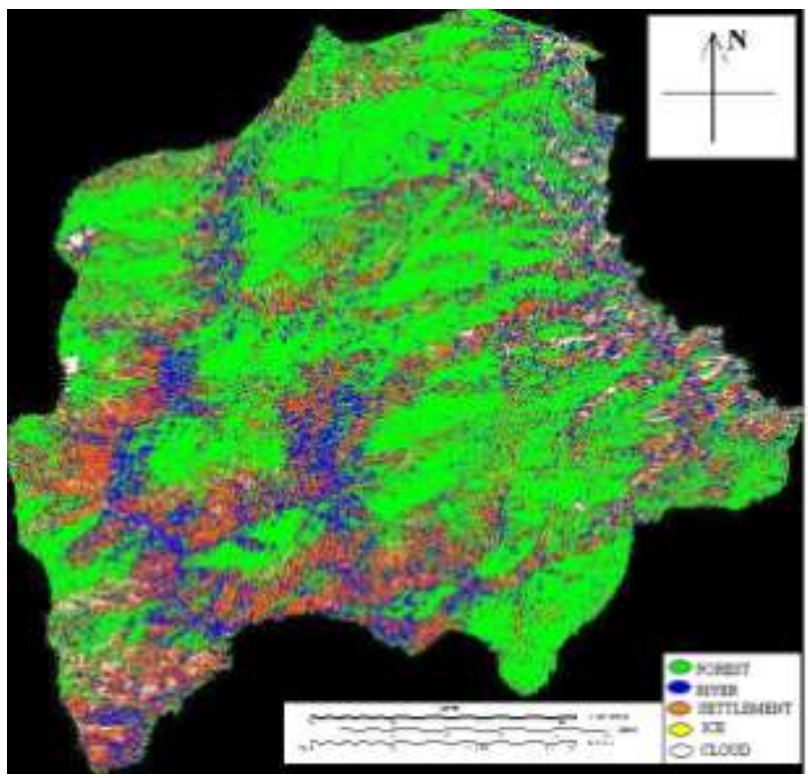

Figure 2: Classified image based on Hybrid classifier.

Table 1: Confusion Matrix for Bayesian Classification.

\begin{tabular}{|c|c|c|c|c|c|c|}
\hline \multicolumn{7}{|c|}{ REFERENCE DATA } \\
\hline & Cloud & Forest & Ice & River & $\begin{array}{c}\text { Settle- } \\
\text { ment }\end{array}$ & $\begin{array}{c}\text { Row } \\
\text { Total }\end{array}$ \\
\hline Cloud & 483 & 0 & 0 & 0 & 0 & 483 \\
\hline Forest & 14 & 130 & 15 & 7 & 3 & 169 \\
\hline Ice & 2 & 1 & 187 & 0 & 3 & 193 \\
\hline River & 0 & 5 & 0 & 113 & 18 & 136 \\
\hline $\begin{array}{c}\text { Settlement } \\
\text { Column } \\
\text { Total }\end{array}$ & 505 & 145 & 202 & 137 & 68 & 1057 \\
\hline
\end{tabular}

Table 2: Error and accuracy for Bayesian Classification.

\begin{tabular}{|c|c|c|c|c|}
\hline Classes & $\begin{array}{c}\text { Commission } \\
\text { Error } \\
(\%)\end{array}$ & $\begin{array}{c}\text { Omission } \\
\text { Error } \\
(\%)\end{array}$ & $\begin{array}{c}\text { Producer's } \\
\text { Accuracy } \\
(\%)\end{array}$ & $\begin{array}{c}\text { User's } \\
\text { Accuracy } \\
(\%)\end{array}$ \\
\hline Cloud & 0 & 5.66 & 95.64 & 100 \\
\hline Forest & 23.08 & 10.34 & 89.66 & 76.92 \\
\hline Ice & 3.11 & 7.42 & 92.57 & 96.89 \\
\hline River & 16.91 & 17.52 & 82.48 & 83.09 \\
\hline Settlement & 42.11 & 35.29 & 64.71 & 57.89 \\
\hline
\end{tabular}


To assess accuracy of the proposed technique, the confusion matrix along with the errors of omission and commission and producer and user accuracy for Bayesian Classifier is given in Table 1 and Table 2 respectively. For hybrid classifier the confusion matrix along with the errors of omission and commission and producer and user accuracy for Bayesian Classifier is given in Table 3 and Table 4 respectively. The Overall Accuracy of Bayesian Classifier and Hybrid classifier are $90.53 \%$ and $91.59 \%$ respectively.

Table 3.6: Confusion Matrix for Hybrid Classification.

\begin{tabular}{|c|c|c|c|c|c|c|}
\hline \multicolumn{7}{|c|}{ REFERENCE DATA } \\
\hline & C & F & I & R & S & $\begin{array}{c}\text { Row } \\
\text { Total }\end{array}$ \\
\hline Cloud & 483 & 0 & 0 & 0 & 0 & 483 \\
\hline Forest & 16 & 139 & 8 & 2 & 4 & 169 \\
\hline Ice & 7 & 0 & 207 & 2 & 2 & 218 \\
\hline River & 0 & 14 & 0 & 113 & 9 & 136 \\
\hline \begin{tabular}{c} 
Settlement \\
\hline $\begin{array}{c}\text { Column } \\
\text { Total }\end{array}$
\end{tabular} & 512 & 171 & 216 & 119 & 64 & 1082 \\
\hline
\end{tabular}

Table 3.7: Error and accuracy for Hybrid Classification.

\begin{tabular}{|c|c|c|c|c|}
\hline Classes & $\begin{array}{c}\text { Commission } \\
\text { Error (\%) }\end{array}$ & $\begin{array}{c}\text { Omission } \\
\text { Error }(\%)\end{array}$ & $\begin{array}{c}\text { Producer's } \\
\text { Accuracy } \\
(\%)\end{array}$ & $\begin{array}{c}\text { User's } \\
\text { Accuracy } \\
(\%)\end{array}$ \\
\hline Cloud & 0 & 5.66 & 94.33 & 100 \\
\hline Forest & 17.75 & 18.71 & 81.28 & 82.24 \\
\hline Ice & 5.04 & 4.16 & 95.83 & 94.95 \\
\hline River & 16.91 & 5.04 & 94.95 & 83.08 \\
\hline Settlement & 35.52 & 23.43 & 76.56 & 64.47 \\
\hline
\end{tabular}

\section{SUMMARY AND CONCLUSION}

In this paper, a Bayesian classifier and hybrid classifier algorithm for remotely sensed satellite data has been developed. To test and validate the algorithm, the sample image taken into consideration is multi-spectral IRS-1C/LISS III of East Sikkim, India. The proposed classifier can also be used for hyperspectral remote sensing data considering the best bands as input for preparing spectral class distribution. The sample image is classified by both Bayesian and Hybrid classifier and then the overall accuracy were calculated. The overall accuracy for the sample test image was found to be $90.53 \%$ using the Bayesian classifier and $91.59 \%$ using the Hybrid respectively. The reason for high accuracy may be to some extent attributed for the reason that the part of the training set is being considered as ground truths instead of actual data. Since the accuracy of the results depends only upon the test set chosen, the efficiency of any algorithm shall not be considered on the accuracy measure alone. The classified images shall also be compared ground truth information physically. From the comparison, it is found that both the methods are equally efficient.

\section{ACKNOWLEDGMENT}

We would like to thank All India Council for Technical Education (AICTE) for funding the project title "Contour Mapping and 3D Surface Modeling of State Sikkim" fully sponsored by All India Council of Technical Education, Govt. of India vide order no- 8023/BOR/RID/RPS-44/2008-09. We also like to thank my fellow students - Bhanu, Khushboo and Prerna for their invaluable support.

\section{REFERENCES}

[1] Tzotsos A. and Argialas D. 2008. Support Vector Machine Classification for Object-Based Image Analysis, LNGC, pp. 663-667.

[2] Gigandet X. et al. 2005. Region-Based Satellite Image Classification: Method and Validation, Proc. of IEEE International Conference on Image Processing, pp. 832835

[3] Friedl, M.A., and Brodley, C.E., 1997. Decision tree classification of land cover from remotely sensed data, Remote Sensing of Environment, Vol. 61, pp 399-409.

[4] Mahesh Pal, and Paul M. Mathers, 2001. Decision Tree Based Classification of Remotely Sensed Data, 22nd Asian Conference on Remote Sensing.

[5] Mahesh Pal, and Paul M. Mathers, 2003. An assessment of the effectiveness of decision tree methods for land cover classification, Remote Sensing of Environment, Vol. 86, pp $554-565$.

[6] Gorte, B. and Stein, A. 2002. Bayesian classification and class area estimation of satellite images using stratification, IEEE Transactions on Geoscience and Remote Sensing, pp. 803-812.

[7] LAU C.C. and Hsiao K.H. (2002). Bayesian Classification for rice paddy interpretation, in proc. of Asian Conference on Remote Sensing, p 140.

[8] Jeliazkova N., Ruseva S., and Boyanov K. (2006). Application of nonparametric Bayesian classifier to remote sensing data.

[9] Perea1 J. A., Meroño1 J. E., and Aguilera1 M. J. (2009). Algorithms of Expert Classification applied in Quickbird satellite images for Land use mapping, Chilean journal of agricultural research, Vol. 69, No. 3, pp. 400-405.

[10] Besag, J., J. York, and A. Mollie. 1991. Bayesian image restoration, with two applications in spatial statistics (with discussion). Annals of the Institute of Statistical Mathematics Vol. 43, pp.1- 59.

[11] Earl Gose, Richard Johnsonbaugh and Steve Jost (1996), Pattern recognition and image analysis, USA. 